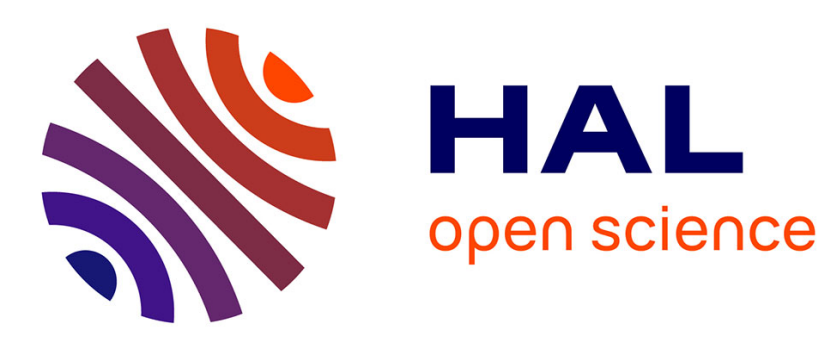

\title{
La recherche française et la discrimination ethnico-raciale dans l'orientation scolaire: un problème de point de vue
}

Fabrice Dhume

\section{- To cite this version:}

Fabrice Dhume. La recherche française et la discrimination ethnico-raciale dans l'orientation scolaire: un problème de point de vue. Journal du Droit des Jeunes. Revue d'Action Juridique et Sociale, 2011, L'école lieu de souffrance.. et de solutions?, 308, p.28-32. halshs-01421970

\section{HAL Id: halshs-01421970 \\ https://shs.hal.science/halshs-01421970}

Submitted on 23 Dec 2016

HAL is a multi-disciplinary open access archive for the deposit and dissemination of scientific research documents, whether they are published or not. The documents may come from teaching and research institutions in France or abroad, or from public or private research centers.
L'archive ouverte pluridisciplinaire HAL, est destinée au dépôt et à la diffusion de documents scientifiques de niveau recherche, publiés ou non, émanant des établissements d'enseignement et de recherche français ou étrangers, des laboratoires publics ou privés. 
Dhume Fabrice, "La recherche française et la discrimination ethnico-raciale dans l'orientation scolaire : un problème de point de vue ", Journal du droit des jeunes, $n^{\circ} 308$ ("L'école lieu de souffrance... et de solutions ? "), p.28-32.

\section{La recherche française et la discrimination ethnico-raciale dans l'orientation scolaire : un problème de point de vue}

Fabrice DHUME, sociologue, chercheur à l'ISCRA - www.iscra.org

En matière de discriminations ethnico-raciales, la recherche française en sciences sociales a surtout mis l'accent sur le domaine du travail et de l'emploi, depuis les travaux inauguraux des années 1990. Début 2000, le champ du logement a été régulièrement intégré dans la littérature de synthèse qui fait le tour d'horizon du phénomène ${ }^{1}$. Pendant ce temps, la problématique de la discrimination à l'école, et plus spécifiquement dans l'orientation et les parcours scolaires, est demeurée quasi informulée et inexplorée. Comment comprendre cela ? Et quel est en réalité l'état de la recherche en la matière ?

\section{Un retour analytique et critique sur l'état de la littérature}

Une revue de la littérature française, réalisée par l'Institut social et coopératif de recherche appliquée (ISCRA) à la demande de la HALDE et l'ACSE ${ }^{2}$, fait le point sur les connaissances en même temps qu'elle rend compte de l'état de la reconnaissance politico-scientifique de l'hypothèse d'une discrimination selon "l'origine " dans l'orientation scolaire ${ }^{3}$. Ce travail repose sur l'analyse d'un corpus de 465 documents, publiés entre 1964 et 2010. Peu de travaux s'inscrivent spécifiquement à l'intersection des trois notions : "orientation scolaire » et « discrimination » selon " l'origine (étrangère) ». Ces notions sont effectivement dotées de traditions épistémologiques distinctes, non articulées entre elles, et inégalement pourvues de légitimité scientifique. Si d'un côté l'orientation scolaire est l'objet d'une abondante littérature, de l'autre les dictionnaires spécialisés ne retiennent même pas l'entrée "discrimination $»^{4}$. Plus encore, ni l'indexation dans les bases de données, ni les titres des travaux de recherche ne permettent d'identifier de prime abord une littérature répondant à la question : y a-t-il en

1 Ex: SLAMA S. (dir.) (2009), Les discriminations selon l'origine, "Problèmes politiques et sociaux " $n^{\circ} 966$, Paris, La Documentation française; RICHARD J.-L. (dir.) (2005), Les immigrés dans la société française, «Problèmes politiques et sociaux » $n^{\circ} 916$, Paris, La Documentation française.

2 Haute autorité de lutte contre les discriminations et pour l'égalité ; Agence nationale pour la cohésion sociale et l'égalité des chances.

3 DHUME F., DUKIC S., CHAUVEL S., PERROT P. (2011), Orientation scolaire et discrimination. De l'(in)égalité de traitement selon "'origine", Paris, La Documentation française.

4 VAN ZANTEN A. (dir.) (2008), Dictionnaire de l'éducation, Paris, PUF, « Quadrige »; BARREAU J.-M. (dir.) (2007), Dictionnaire des inégalités scolaires, Paris, ESF éditeur. 
France de la discrimination selon "l'origine " dans l'orientation des élèves ? Formellement, cette question semble inédite, comme cela avait déjà été noté ${ }^{5}$.

A contrario, il existe une foisonnante littérature - et près d'une dizaine de revues bibliographiques ou de synthèses depuis 1977 - articulant telles des instances " école » et « immigration $\|^{6}$. Cette littérature est, dès le début, marquée par la thèse de la " distance » (culturelle, sociale, morale) entre certains publics et l'école. Construite en référence aux "pensées d'Etat ${ }^{7}$ que sont l'immigration et l'école, la plupart de ces travaux semble ignorer la question de la discrimination, et développe des théories finalement proches de la norme du pouvoir. Ce constat rejoint celui de Jean-Paul Briand et Jean-Michel Chapoulie, selon qui les études sur les inégalités dans le système éducatif privilégient systématiquement une approche par les publics scolarisés, au détriment de celles en termes de logiques institutionnelles ${ }^{8}$. A partir d'une analyse critique et politique de l'état de la recherche, on verra que les problèmes de (re)connaissance des discriminations dans l'orientation scolaire ont largement trait à cette question du point de vue développé sur l'école.

\section{Deux points de vue antagoniques et trois gammes de travaux}

A la question de savoir s'il y a de la discrimination selon " l'origine » dans l'orientation et les parcours scolaires, les sciences sociales répondent de façon diamétralement contradictoire. La majeure partie de la littérature pointe vers l'idée que " l'école intègre et ne discrimine pas " - selon la formule de Jean-Luc Richard ${ }^{9}$; mais d'autres chercheurs montrent que des élèves subissent une inégalité de traitement et une disqualification scolaire relatives à leur statut ethnico-racial ${ }^{10}$. Dès les années 1970 , le champ de la recherche a vu cohabiter ces deux points de vue antagoniques, avant qu'une troisième gamme de travaux n'émerge. Précisons la perspective et la position de chacune de ces trois gammes de travaux.

Une gamme majoritaire. Celle-ci a donné les premières études consacrées spécifiquement aux « enfants d'immigrés » à l'école ${ }^{11}$. Elle est dominante dans le champ en nombre d'écrits (la moitié du corpus) et en reconnaissance (diffusion des thèses, citation des articles, etc.). Centrée sur le public scolarisé, elle combine au départ la socio-démographie et une

5 NAVES M.-C. (2002), Les discriminations raciales à l'école, Paris, ADRI/Programme RAXEN.

6 LAACHER S. (2005), L'institution scolaire et ses miracles, Paris, La Dispute.

7 SAYAD A. (1999), «Immigration et "pensée d'Etat" ", Actes de la recherche en sciences sociales, n¹29, p.514.

8 BRIAND J.-P., CHAPOULIE J.-M. (1993), "L'institution scolaire et la scolarisation: une perspective d'ensemble », Revue Française de sociologie, n³4, p.3-42.

9 RICHARD J.-L. (1999), "L'école intègre et ne discrimine pas ». [En ligne] URL : http://census.web.ined.fr/ debat/Contributions/Mars-1999/listcensus163j.html,

10 Cette notion insiste sur le fait que l'ethnique et le racial ne sont pas des propriétés primordiales des individus ou des groupes, mais des rapports sociaux assignant à certains un statut d'altérité et de minorité relatif à une " origine » ou à une « nature » présumée différente.

11 Premiers travaux : CLERC P., GIRARD A. (1964), « Nouvelles données sur l'orientation scolaire au moment de l'entrée en sixième ", Population, vol.19, n5, p.829-872 ; LAMBIOTTE-FEKKAR B. (1966), "Le problème de l'adaptation scolaire des enfants algériens de la région parisienne », Enfance, $n^{\circ} 4-5$, p.129-136 ; DESCLOITRES R., FAYARD D. (1970), L'apprentissage social des écoliers algériens à Aix-en-Provence, Centre des sciences humaines appliquées. 
psychologie aux accents culturalistes. La catégorie de l'« origine » y apparaît sur un mode "primordialiste $~^{12}$, c'est-à-dire comme une propriété des individus existant antérieurement aux interactions sociales. Ils cherchent alors à déterminer si oui ou non, et comment, " l'origine » des élèves ou des familles influence l'adaptation scolaire, les performances et la place des élèves dans l'école. Cette gamme est qualifiée de majoritaire du fait de sa dominance dans le champ et parce qu'elle représente le point de vue de "Nous » (le Majoritaire, le Normal) sur « Eux » (les minorisés).

Une gamme minoritaire. Avec sa dominante de sociologie empirique et de psychosociologie, cette gamme se caractérise par la mise en question de l'action de l'école ou les interactions autour d'elle. Dès le milieu des années $1970^{13}$, ces travaux mettent en évidence l'influence de stéréotypes dans le jugement enseignant et l'importance des catégories ethnico-raciales dans les relations et rapports scolaires. Ce point de vue tient " l'origine " pour un élément des interactions entre l'école et ses publics, à la fois stigmate (objet de préjugés, etc.) et ressource. Souvent plus sensible au vécu des publics et à leur expérience de l'école, cette gamme peut être dite minoritaire, d'autant qu'elle a longtemps été marginalisée dans le champ.

Une gamme intermédiaire. Suivant la décentralisation du système scolaire, une troisième gamme de travaux sociologiques, économiques et politologiques émerge vers $1990^{14}$. Avec son référent socio-géographique, elle parle territoires, effets de contexte, zones d'éducation prioritaire, etc. Suivant le jeu de bonneteau de la « discrimination positive à la française », la catégorie ethnique est transférée dans celle de territoire ; l'idée de ségrégation signale la contribution de cette gamme à la reconnaissance d'une question ethnico-raciale. Gamme intermédiaire, elle ouvre un espace de recherche d'ampleur croissante, qui alimente, étaye, articule ou infléchit l'une et l'autre gammes précédentes.

Comment comprendre que la discrimination ethnico-raciale dans l'orientation scolaire soit si peu reconnue, alors que ce terme est employé dès 1978 par Jean-Pierre Zirotti ${ }^{15}$ ? Et surtout alors qu'une accumulation de travaux qualitatifs, plus que dans tout autre secteur de l'action publique ${ }^{16}$, pointe la responsabilité de l'institution scolaire et de ses agents dans les processus d'ethnicisation. Comment se fait-il, aussi, que malgré des efforts répétés de

12 POUTIGNAT P., STREIFF-FENART J. (1999), Théories de l'ethnicité, Paris, PUF, p.99.

13 Premiers travaux: AMIGUES R., BONNIOL J.-J., CAVERNI J.-P. (1975), « Les comportements d'évaluation dans les systèmes éducatifs. Influence d'une catégorisation ethnique sur la notation de productions scolaires ", International journal of psychology, vol.10, $\mathrm{n}^{\circ}$ 2, p.135-145; ZIMMERMANN D. (1978), " Un langage non-verbal: les processus d'attraction-répulsion des enseignants à l'égard des élèves en fonction de l'origine familiale de ces derniers ", Revue française de pédagogie, $\mathrm{n}^{\circ} 44, \mathrm{n}^{\circ} 1$, p.46-70; NOVI M., ZIROTTI J.-P. et alii (1979), La scolarisation des enfants de travailleurs immigrés, t.1 et t.2, Nice, IDERIC.

14 Premiers travaux : LEGER A., TRIPIER M., non daté [1983-84], Echec scolaire et cohabitation multi-ethnique dans un quartier populaire "Les Grésillons", Centre de recherche et d'études sur la société française ; PAYET J.-P. (1992), " Civilités et ethnicité dans les collèges de banlieue : enjeux, résistances et dérives d'une action scolaire territorialisée », Revue française de pédagogie, $n^{\circ} 101$, p.59-69; BROCCOLICHI S. (1995), "Orientations et ségrégations nouvelles dans l'enseignement secondaire ", Sociétés contemporaines, $\mathrm{n}^{\circ} 21, \mathrm{p} .15-27$.

15 ZIROTTI J.-P. (1978), « Une enquête sur l'orientation scolaire des adolescents immigrés », Migrants-Formation, n²9-30, p.77-82.

16 LORCERIE F. (2007), "Le paradigme de l'ethnicité. Développements en France et perspectives » Faire Savoirs, $n^{\circ} 6$, p.15-23. 
structuration du domaine de savoir par des auteurs ayant inauguré ces questions ${ }^{17}$, l'idée de discrimination semble encore si difficile à faire admettre ? Je proposerai trois séries d'arguments, dont la première tient à la dynamique politique et aux rapports de force qui structurent le champ scientifique.

\section{Rapports de force et dynamique politique du champ scientifique}

L'idée de gammes majoritaire et minoritaire indique des différences de statut dans les rapports de pouvoir ; car si l'on admet l'égale validité scientifique des divers travaux, il faut bien voir qu'ils n'ont pas la même légitimité ; certains dominent. La dominance de la gamme majoritaire s'accompagne de plusieurs problèmes théoriques, dont en voici au moins quatre. Premièrement, l'un des arguments centraux de ces travaux tient à une opposition entre " ethnique » et « social »; si les enfants étrangers sont " en échec », disaient par exemple S. Boulot et D. Boyzon-Fradet, cela tient "à leur qualité de fils d'ouvrier ${ }^{18}$. Politiquement parlant, ce discours des années 1980 est une réponse à la montée de l'extrême-droite (on déspécifie l'immigration face à sa stigmatisation) et une manière de rembrayer l'analyse des rapports de classes. Bien entendu, ces derniers contribuent largement à organiser et justifier la discrimination scolaire. Mais, le poids des théories marxistes a joué de façon contradictoire, favorisant d'un côté la reconnaissance de la (re)production d'inégalités sociales par l'école, et bloquant d'un autre côté la reconnaissance de rapports de domination de sexe et de race, notamment. Or, il y a des raisons de penser que divers motifs de disqualification sont à l'oeuvre et s'entremêlent. Par ailleurs, les catégories dites sociales sont si banales dans la pensée sociologique qu'elles semblent tenues pour naturelles voire « universelles ${ }^{19}$. Deux effets paradoxaux en découlent : non seulement, les catégories sont substantivées $^{20}$, comme si le social était en soi évident et limpide, tandis que l'ethnique poserait en soi problème ; mais aussi, on fait comme si le social et l'ethnique étaient d'une nature différente, et donc comme si le second n'était pas également une catégorie sociale...

Deuxièmement, la gamme majoritaire déploie une façon singulière de poser les problèmes : on y explique la particularité de la position scolaire souvent dégradée (échec, décrochage, surreprésentation dans l'éducation spécialisée ou les filières professionnelles, etc.) du public « issu de l'immigration » par une spécificité qui lui est attribuée. Les travaux sur la réussite, à la fin des années 1980, n'ont pas changé cette logique: la réussite y est qualifiée de

17 ZIROTTI J.-P. (1989), « Constitution d'un domaine de recherche : la scolarisation des enfants de travailleurs immigrés (ETI) », Babylone, n6-7, p.210-254; LORCERIE F. (1995), « Scolarisation des enfants d'immigrés. Etat des lieux et état des questions en France », Confluence Méditerranée, n¹4, p.27-66 ; PAYET J.-P. (1996), « La scolarisation des enfants et des jeunes issus de l'immigration en France », Revue française de pédagogie, $n^{\circ} 117$, p.87-149 ; etc.

18 BOULOT S., BOYZON-FRADET D. (1984), « L'échec scolaire des enfants d'immigrés. Un problème mal posé », Les Temps modernes, $\mathrm{n}^{\circ}$ spécial, 452-453-454, p.18-31

19 D. Schnapper justifie ainsi son refus d'utiliser "des critères ethniques », intervention au séminaire national : « Lutter contre les discriminations : la diversité à l'Ecole », MEN, Paris, 7 octobre 2009.

20 LATOUR B. (2006), Changer de société, refaire de la sociologie, Paris, La Découverte. 
" paradoxale » ou "surprenante », et la causalité est recherchée dans la " mobilisation familiale » et la "volonté d'intégration ${ }^{21}$. Comme si dans l'opération de tri et de classement social qu'est le processus d'orientation, le rôle de l'école était neutre, constant ou secondaire et que seule la qualité du public était déterminante.

Troisième problème: la gamme majoritaire semble la plus institutionnellement installée, légitimée et diffusée. Les travaux parmi les plus fameux et les plus souvent cités du corpus ${ }^{22}$ peuvent être issus du ministère de l'Education nationale ou d'autres organismes d'Etat. Si cela ne met pas en doute leur pertinence, on peut s'interroger sur le rapport entre cette situation et la sorte d'évidence du point de vue de l'institution scolaire - la normalité de sa norme, pour ainsi dire, qui semble traverser nombre de recherches. Cette " hiérarchie de crédibilité » en faveur de l'institution ${ }^{23}$ peut donner à la thèse d'une absence de discrimination le poids d'un savoir institué, et permettre par exemple de renvoyer le discours d'expérience de la discrimination au rang vulgaire des "faits divers et (de) l'indignation ${ }^{24}$. Quatrièmement, la gamme majoritaire s'appuyant très largement sur les méthodes statistiques, elle peut parfois jouer d'un effet d'autorité que lui confère la dominance des modèles économétriques qui se sont imposés dans les années 1990, au point, parfois, de présenter ces méthodes comme les seules valables pour démontrer (ou invalider l'hypothèse de) la discrimination ${ }^{25}$. Comme le note Philippe Perrot, si les implicites de ce raisonnement sont admis tels quels, "la recherche sociologique peut difficilement parler de discriminations ${ }^{26}$.

\section{Une notion politiquement récente et scientifiquement peu investie}

La difficulté à reconnaître la discrimination trouve aussi des raisons dans l'état du champ politique, et dans la proximité de la recherche avec celui-ci. L'usage du concept de " discrimination raciale » pour qualifier la situation française est récent : on peut dater de 1998 l'inflexion officielle du discours gouvernemental. Dans les titres du corpus, le terme de "discrimination " n'apparaît explicitement qu'après cette date, et d'abord à travers un rapport public du Haut conseil à l'intégration $(\mathrm{HCl})^{27}$. Les sciences sociales vont suivre le mouvement: 13\% des titres parus après 1998 emploient désormais le terme. Cette reconnaissance tardive s'est faite en grande partie à la lumière politique des « émeutes de

21 ZEROULOU Z. (1988), « La réussite scolaire des enfants d'immigrés : l'apport d'une approche en termes de mobilisation », Revue française de sociologie, $n^{\circ} 88$, p.447-470.

22 Notamment : VALLET L.-A., CAILLE J.-P. (1996), « Les élèves étrangers ou issus de l'immigration dans l'école et le collège français. Une étude d'ensemble », Les dossiers d'Education et formations, $n^{\circ} 76, \mathrm{MEN}$.

23 BECKER H. (2002), Les ficelles du métier, Paris, La Découverte, p.152-155.

24 C'est le propos de : DUBET F. (1989), Immigrations : qu'en savons-nous ? Un bilan des connaissances, Paris, La Documentation française, p.51.

25 Selon Marie Duru-Bellat, l'étude de la discrimination impliquerait a priori une approche par les « modèles multivariés » et à la méthode dite des "résidus ». DURU-BELLAT M. (2002), Les inégalités sociales à l'école. Genèse et mythes, Paris, PUF, p.44.

26 PERROT P. (2006), " La discrimination systémique dans le système éducatif français », Cahiers de I'URMIS, $\mathrm{n}^{\circ} 10-11$. [En ligne] URL : http://urmis.revues.org/index259.html.

$27 \mathrm{HCl}$ (1998), Lutter contre les discriminations : faire respecter le principe d'égalité, Paris, La Documentation française. 
$2005 »^{28}$ - ce qui est assez paradoxal, car contrairement à des mouvements précédents les " émeutiers » ont peu argué de la discrimination, et c'est surtout le gouvernement qui a instrumentalisé ce thème. La proximité de la recherche avec l'agenda politique et avec l'institution scolaire rend artificielle une séparation des arènes du débat scientifique, institutionnel et social-politique. Cette situation peut en revanche avoir pour conséquence un faible investissement scientifique de l'idée de discrimination. Et d'abord au plan conceptuel : souvent simple emprunt au discours commun de l'époque, le terme s'avère rarement défini précisément, et quasiment jamais investi d'un soubassement théorique explicite. II requalifie des schèmes interprétatifs précédents sans en infléchir le sens, servant par exemple à rembrayer des analyses en termes de "ségrégation ${ }^{29}$, de «manque de capital symbolique $~^{30}$; ou encore, il est tenu pour simple euphémisation "d'un racisme, plus élégamment nommé discrimination $»^{31}$. Autant de signes que la diffusion du terme dans la littérature ne coïncide pas avec un renouvellement conceptuel.

Le malentendu se prolonge d'autant que les schémas théoriques de la gamme majoritaire sont souvent très en phase avec les référentiels de politique publique. Dès 1970, les sciences sociales ont emboîté le pas à une politique ministérielle qui a construit les " enfants d'immigrés » comme problème pour l'école. Aujourd'hui aussi, nombreux sont les travaux qui sont tournés vers des approches par l'insertion et l'intégration, et/ou par l'interculturel et le racisme, suivant les priorités politiques. La prégnance de ces référentiels conduit presque toujours à minorer le phénomène discriminatoire ou à réduire la portée heuristique du concept. Par exemple, la référence usuelle au racisme conduit des chercheurs à définir la discrimination de façon restrictive, comme " séparation volontaire " ou action " délibérée ${ }^{32}$. Cela montre au passage un cloisonnement du champ, les auteurs semblant ignorer les nombreux travaux sur le rôle des intermédiaires à l'emploi, qui ont de longue date montré le caractère coproduit d'une discrimination dite "systémique » ou en réseau ${ }^{33}$. Autre exemple : la logique d'insertion conduit les sciences sociales à reconnaître un effet de détermination de positions sociales des publics «issus de l'immigration » par l'école; mais souvent, ces publics ne sont tenus pour discriminés que sur le marché du travail ${ }^{34}$; l'école est rarement caractérisée comme discriminatoire. Se prolonge ainsi l'idée, imprimée dès 1998 par le $\mathrm{HCl}$, selon laquelle « en règle générale, les trajectoires scolaires des jeunes issus de l'immigration

28 La référence est explicite chez : OBERTI M. (2007), L'école dans la ville. Ségrégation, mixité, carte scolaire, Paris, Presses de Science Po; CASTEL R. (2007), La discrimination négative. Citoyens ou indigènes ?, Paris, Seuil/La République des idées ; DUBET F. (2008), La galère : jeunes en survie, Paris, Points (Préface).

29 VAN ZANTEN A. (2006), « Une discrimination banalisée ? L'évitement de la mixité sociale et raciale dans les établissements scolaires ", in Fassin D., Fassin E. (dir.), De la question sociale à la question raciale. Représenter la société française, Paris, La Découverte, p.195-210.

30 BERTHET T. (2006), "Discriminations dans les mondes de l'éducation et de la formation : regards croisés », Relief, n¹7, CEREQ.

31 CHOMENTOWSKI M. (2009), L'échec scolaire des enfants de migrants. L'illusion de l'égalité, Paris, L'Harmattan.

32 Respectivement: BEN AYED C. (2011), "Discriminations : l'éducation un espace à haut risque ? ", Le sociographe, $n^{\circ} 34$; OBERTI M. (2007), L'école dans la ville, op. cit., p.254.

33 Voir notamment les travaux de I'ISCRA, de I'URMIS, de I'IRES sur ce point...

34 Par exemple : RICHARD J.-L. (1999), « L'école intègre et ne discrimine pas », op. cit. ; LAINE F., OKBA M. (2005), « L'insertion des jeunes issus de l'immigration : de l'école au métier », Net.doc, $\mathrm{n}^{\circ} 15$, CEREQ. 
ne témoignent pas de discrimination significative $\aleph^{35}$, contrairement à d'autres secteurs tel l'emploi. Sans plus de regard sur les différences de données disponibles (le champ scolaire n'a pas de statistiques équivalentes à celles du marché du travail), ni sur les singularités sectorielles (la sélection scolaire diffère de celle de l'emploi ou du logement), on fait comme s'il y avait une rupture essentielle de l'ordre social coïncidant avec les frontières de l'institution. Ce qui n'est en réalité pas tenable ${ }^{36}$.

\section{Réticence idéologique et résistance institutionnelle}

Enfin, il faut voir dans la résistance à envisager ces questions un effet de la réticence française à reconnaître et conceptualiser les rapports ethnico-raciaux ${ }^{37}$. Marquée du sceau idéologique d'un tabou "républicain » et antiraciste, a fortiori dans le " sanctuaire " qu'est censée être "l'Ecole de la République », la question de la discrimination raciale a longtemps été confinée au statut d'impensable et d'innommable. Dans la littérature, cela se traduit d'au moins deux manières : d'une part ces questions et concepts sont assimilés à des produits d'importation issus "des modèles américains et sud-africains ${ }^{38}$; d'autre part, les manifestations d'ethnicité et de racisme sont traitées comme « un phénomène social extrinsèque à l'Ecole ${ }^{39}$. On fait donc comme si le phénomène et son sens s'imposaient de l'extérieur à une société française soumise à la mondialisation des normes culturelles " anglo-saxonnes", et aussi à une institution scolaire subissant l'irruption de nouveaux publics indésirables. Ce faisant, les sciences sociales semblent souvent entretenir un lien complexe avec l'idéologie républicaine, diffusant l'idée d'une « spécificité du modèle français d'intégration ». F. Lorcerie a montré que divers auteurs ont significativement contribué au retour de la question de "l'identité nationale " sur la scène politique, dans les années $1990^{40}$. Une partie de la recherche a en tout cas pu trouver dans le mot d'ordre politique de "l'intégration » une raison suffisante pour « expliquer » les trajectoires scolaires des élèves « issus de l'immigration ».

Les façons de nommer les problèmes témoignent de la relation compliquée des sciences sociales aux questions ethnico-raciales. Alors que le concept d'ethnicité désigne conventionnellement les processus de production et d'attribution d'" origines " dans les interactions sociales, les chercheurs hésitent à l'employer, ou alors ils le font en oubliant le cadre théorique interactionniste de cette notion. Ils préfèrent le plus souvent l'idée

$35 \mathrm{HCl}$ (1998), Lutter contre les discriminations, op. cit., p.25.

36 DHUME F. (2011), Entre l'école et l'entreprise, la discrimination ethnico-raciale dans les stages. Une sociologie publique de l'ethnicisation et de l'ordre scolaires, Thèse de doctorat de sociologie, Université d'Aix-en-Provence.

37 STREIFF-FENART J. (2002), « Un dilemme français : modèle républicain et discriminations ethniques », Faire Savoirs, $\mathrm{n}^{\circ} 1$, p.69-76.

38 BARTHON C. (1997), "Enfants d'immigrés au collège : intégration ou ségrégation scolaire ? », in Aubert F., Tripier M., Vourc'h F. (dir.), Jeunes issus de l'immigration. De l'école à l'emploi, Paris, Ciemi/L'Harmattan, p.97.

39 TAPERNOUX P. (1997), Les enseignants face aux racismes, Paris, Anthropos, p.37. Cf. aussi DUBET F., DURU-BELLAT M. (2002), " Les problèmes sociaux entrent dans l'école », in Carra C., Faggianelli D.(dir.) Ecole et violences, « Problèmes politiques et sociaux " $n^{\circ} 881$, Paris, La documentation française.

40 LORCERIE F. (1992), « Les sciences sociales au service de l'identité nationale. Le débat sur l'intégration en France au début des années 1990 », in Martin D.-C. (dir.), Cartes d'identité. Comment dit-on « nous » en politique ?, Presses de la Fnsp, p.245-281. 
d'« origines », avec sa connotation d'identité objective et primordiale, et avec ses ambiguïtés - le terme accueille indistinctement des usages juridiques, ethnico-raciaux, généalogiques et géographiques. Les stratégies discursives indiquent parfois la conscience et une prudence à l'égard des effets d'assignation des catégories (par exemple l'emploi abondant des guillemets), mais elles montrent en même temps le rapport fort problématique des sciences sociales avec ces questions ${ }^{41}$. C'est là le fruit d'une "double contrainte ${ }^{42}$ pesant sur le champ intellectuel (et linguistique) français : une hésitation ou une tension entre nommer (et performer) et innommer (et occulter) le référent ethnico-racial. Le terme de " discrimination » étant perçu comme intrinsèquement lié à l'ethnico-racial ${ }^{43}$, son éviction des questions de la recherche peut aussi témoigner de ce malaise.

Au sein du champ scientifique français, la question de l'immigration et des rapports ethnicoraciaux fait partie de celles, minorisées, selon une homologie avec le statut des populations concernées $^{44}$; mais à l'intérieur même de cette question se joue la minorisation de certains savoirs. Celui sur la discrimination à l'école semble bien relever de ce que Michel Foucault nomme des «savoirs assujettis", c'est-à-dire «disqualifiés comme savoirs (...) hiérarchiquement inférieurs $»^{45}$. C'est pourquoi reconnaître et comprendre la discrimination scolaire, dans la subtilité de ses processus, suppose de réinterroger les points de vue mêmes de la recherche.

41 DHUME F. (2010), " De la race comme un problème. Les sciences sociales et l'idée de nature », Raison présente, $\mathrm{n}^{\circ} 174, \mathrm{p} .53-65$.

42 FELOUZIS G. (2008), « L'usage des catégories ethniques en sociologie. Dossier-débat coordonné et présenté par Georges Felouzis », Revue française de sociologie, vol.49, p.128.

$43 \mathrm{II}$ y a des raisons à cela : les premiers travaux des recherches des années 1990 ont porté principalement sur cela ; et l'Etat français a d'emblée construit sa communication sur la « discrimination raciale ».

44 REA A., TRIPIER M., Sociologie de l'immigration, La Découverte/Repères, 2008.

45 FOUCAULT M. (1997), "II faut défendre la société ». Cours au Collège de France. 1976, Paris, Gallimard/ Seuil, pp.8-9. 